\title{
La recatégorisation comme procédé argumentatif dans le domaine juridique
}

The Mechanism of "Argumentative Recategorization" in the Legal Area

\section{Rubens Damasceno Morais}

\section{OpenEdition}

Journals

\section{Electronic version}

URL: http://journals.openedition.org/aad/1808

DOI: $10.4000 /$ aad. 1808

ISSN: 1565-8961

Publisher

Université de Tel-Aviv

\section{Electronic reference}

Rubens Damasceno Morais, «La recatégorisation comme procédé argumentatif dans le domaine juridique », Argumentation et Analyse du Discours [Online], 13 | 2014, Online since 22 October 2014, connection on 17 June 2020. URL : http://journals.openedition.org/aad/1808 ; DOI : https://doi.org/ 10.4000/aad. 1808

This text was automatically generated on 17 June 2020 .

\section{(c) (i) () $\Theta$}

Argumentation \& analyse du discours est mis à disposition selon les termes de la licence Creative Commons Attribution - Pas d'Utilisation Commerciale - Pas de Modification 4.0 International. 


\title{
La recatégorisation comme procédé argumentatif dans le domaine juridique
}

The Mechanism of "Argumentative Recategorization" in the Legal Area

\author{
Rubens Damasceno Morais
}

\section{Introduction}

1 Cet article explore un aspect particulier de la gestion du désaccord dans le domaine juridique à travers un mécanisme mis en œuvre par des juges brésiliens dans le cadre de leurs délibérations au sein d'une Cour d'Appel. Il s'agit en l'occurrence de la

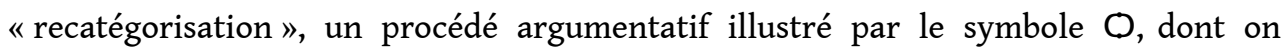
cherchera à décrire ici le fonctionnement et le rôle. C'est, on le verra, grâce à cette stratégie argumentative que les magistrats parviennent à produire des arguments antagoniques dans la bataille des opinions.

2 On explicitera en un premier temps les principes théoriques et méthodologiques qui ont guidé ce travail, pour ensuite clarifier le concept dont on traite ici. Afin d'explorer le mécanisme analysé, on sera amené à décrire le fonctionnement discursif et pragmatique de la stase argumentative ${ }^{1}$, lors des moments de définition d'un fait illicite (entendu ici comme fait générateur d'un préjudice moral susceptible d'être dédommagé), à travers une étude de cas (l'affaire dite "de la file d'attente»). On s'interrogera ensuite sur l'importance de la dimension institutionnelle du contexte des délibérations et ses conséquences sur le système des tours de parole ; sur l'organisation de la conduite des participants de l'interaction (les magistrats); sur la perception subjective, par ces mêmes magistrats, du montant à attribuer au titre de dommages et intérêts; et, enfin, sur l'organisation de la procédure. Plus précisément, on s'intéressera à l'effet qu'ont les contraintes procédurales sur l'interaction, c'est-à-dire à la force du rituel des délibérations dans une Cour d'Appel. A cette fin, on cherchera à rendre compte de quelques-uns des procédés mis en œuvre par les interactants pour 
gérer le désaccord qui surgit entre eux "à vif et à chaud $»^{2}$ dans le cadre des délibérations. On contribuera ainsi à examiner « le droit en action » (Dupret $2006: 19$ ), tout en adoptant un regard de spécialiste du langage. Une telle enquête est d'autant plus importante qu'elle est nécessaire au développement d'une sociologie des réalités juridiques; et elle est d'autant plus intéressante qu'elle concerne des institutions emblématiques et des procédures soumises à controverse, par ailleurs très peu étudiées.

\section{L'activité juridique est avant tout langagière}

3 La représentation de la justice par une femme aux yeux bandés tenant une balance dans la main gauche et un glaive dans la main droite confère à l'espace judiciaire un aspect quelque peu mystérieux, voire mystique (Posner $2008: 3$ ), sacré (Garapon $2001: 38$ ). Elle suggère que cet espace est séparé du reste du monde et toujours pris en charge par des spécialistes (juristes, magistrats, avocats) en perruque, en toge, brandissant un marteau. Par ailleurs, on sait que partout les magistrats, dans l'exercice de leurs fonctions, revêtent une toge. Ils marquent ainsi qu'il s'agit d'un monde hermétique, peuplé de "personnes allégoriques» (Garapon id.: 100), en rupture avec l'espace quotidien des simples mortels. De plus, dans cet univers, «la langue des décisions, des sentences juridiques est naturelle et donc parfois équivoque, voire totalement ambiguë » (Martineau 2010: 403). L'interprétation que donnera le magistrat y est le plus souvent objet de discussions, surtout quand il s'agit de dommages et intérêts, et plus particulièrement de la quantification du pretium doloris (notre domaine actuel de recherche). On dit même que le droit peut être vu comme une "profession of words" (Hiltunen 2010: 61), en raison de l'importance que revêt l'argumentation dans le domaine juridique. C'est pourquoi l'intérêt d'une approche argumentative du champ juridique est difficilement contestable. C'est dans cette perspective que Feteris affirme que les théoriciens de l'argumentation et les théoriciens du droit s'intéressent aux mêmes types de questions (1999: 2).

4 Pour notre part, nous avons constaté, lors des analyses des données à disposition, que les magistrats, dans la plupart des cas, sont supposés justifier leurs choix par des arguments, ce qui montre que "le droit est forcément argumentatif et argumenté " (Meyer 1999: 296). Étant donné que l'argumentation juridique est énoncée dans un cadre qui en prévoit, institutionnellement, l'immédiate contradiction par une argumentation adverse (Martineau $2010: 3$ ), il apparaît qu'un jugement est le résultat d'un type de dialogue, quelquefois polémique, entre les magistrats et les lois (le Code civil, la Constitution) ou entre les magistrats eux-mêmes.

De cette façon, le caractère dialogique, délibératif et argumentatif des débats juridiques, aspirant à la justification rationnelle des solutions avancées, permet aux juges, pendant les débats, de pouvoir toujours être en mesure de soulever des arguments originaux, parfois contraires à ceux qui sont présentés par leurs pairs. C'est en fin de compte ce qui nourrit le monde juridique, car «l'activité juridique est avant tout langagière » (Dupret 2006 : 229). Il est donc pertinent de dépasser le simple regard $\mathrm{du}$ juriste et de porter sur l'argumentation dans le domaine juridique un regard de linguiste, d'analyste des discours, ce qui nous permettra d'identifier quelques procédés argumentatifs significatifs quand il s'agira de montrer comment le dispositif de ce qu'on appelle « recatégorisation » est mis à profit par les magistrats. 


\section{1. « $X$ est un $Y$ en $C$ » : les formules créatrices des faits sociaux}

On le sait, l'établissement des faits pertinents dans le monde juridique présente un relief institutionnel qui les rend a priori complexes, parce qu'il faut toujours établir les faits d'abord pour pouvoir les qualifier ensuite, ou les formuler sous forme de propositions, afin qu'ils puissent constituer une donnée. Cette donnée servira de point de départ au raisonnement, ce qui représente déjà un exercice assez complexe mobilisant une dimension indéniablement normative. Outre la contrainte procédurale, les participants à l'interaction judiciaire en appel s'orientent vers ce qu'on pourrait appeler la pertinence juridique, ce qui «renvoie à l'opération de qualification consistant à faire correspondre une instance factuelle à une définition juridique formelle» (Dupret 2006: 20) - par exemple, déterminer si une action de vol constitue une «infraction» ou un «délit» serait un problème de qualification de droit, nécessitant le recours à des règles très spécifiques. En effet, seule l'argumentation juridique permet d'appliquer une règle à un cas, en montrant que ce cas satisfait aux conditions générales dont la loi fait dépendre l'application de la règle (Cornu $2005: 240)$. En d'autres termes, il faut qu'on reconnaisse " $X$ comme un $Y$ en $C$ » ( $C$ étant le contexte), par une sorte de formule juridique créatrice de faits sociaux. Une telle formule n'a pas pour but d'affirmer une identité entre deux termes, mais d'associer une loi à un fait afin de créer un nouveau fait social. C'est ainsi que Danblon retrace le processus $\mathrm{X}$ est un $\mathrm{Y}$, par lequel il s'agit de qualifier une action dans le domaine strictement juridique (2002: 77).

7 En ce qui concerne la qualification d'une action comme «illicite » au Brésil, surtout dans les affaires de dommages et intérêts en réparation d'un préjudice moral, elle donne toujours lieu à des discussions conséquentes, soit au niveau de la juridiction, soit au niveau doctrinal. La fréquence et l'étendue de ces discussions est sans doute liée au fait que le dommage moral, en raison du degré de polémique des échanges qu'il occasionne, et des conclusions parfois opposées auxquelles arrivent les magistrats au moment de la qualification d'une affaire, est un type de concept flou ${ }^{3}$. Il ne faut en effet jamais oublier que «toute systématisation juridique demeure nécessairement incomplète [et] cela parce que les frontières entre élaboration, interprétation et application du droit ne peuvent jamais être véritablement déterminées » (Vignaux 1979 : 68). Les avis antagonistes prononcés par les magistrats lors des délibérations, ainsi que les revirements de jurisprudence, ne sont possibles qu'à cause de la fluidité même du concept de « dommage moral », lequel pourrait être considéré, à l'image du concept de « justice » lui-même, comme un « fourre-tout discursif »- comme le suggère Angenot (2008: 161). Par ailleurs, pour Perelman et Olbrechts-Tyteca, la justice est une valeur "abstraite» $(2008: 103)$, et la technicité du langage du droit peut être vue comme correspondant à une "démarche de précision" (Cornu 2005, 19), dont l'application peut rendre manifeste des conflits d'appréciation pendant les délibérés, conflits dus à la difficulté de simplement qualifier une action, soit comme «dommage moral " (action illicite entrainant un préjudice), soit comme "simple ennui » (action non-illicite), comme on le verra dans cette analyse. 


\subsection{La recatégorisation argumentative}

jurisprudence), on sait que tout système de droit comporte de nombreux éléments d'incertitude, ce qui donne aux juges une certaine liberté au moment de former leur intime conviction lors de l'établissement des faits, c'est-à-dire au moment de la qualification. Il est très probable que «la personnalité du juge joue un rôle, parfois limité, mais parfois décisif, dans le déroulement du procès et dans son issue " (Perelman 1990 : 590). En raison de la diversité des paramètres qui interviennent dans la décision : valeurs, expérience vécue, normes et principes juridiques, l'émergence de conflits entre les décisions des experts pendant les délibérations devient presque inévitable. Comme l'écrit Perelman à propos du raisonnement juridique (1999:6) : « rares sont les situations où les bonnes raisons, qui militent en faveur d'une solution, ne soient pas contrebalancées par des raisons plus ou moins bonnes en faveur d'une solution différente ».

9 En observant la façon dont les interactants (les magistrats) allient la liberté et la raison au moment de qualifier une affaire en tant que « dommage » - concept ouvert à maintes possibilités d'interprétation juridique au Brésil - il nous est apparu qu'ils utilisent un procédé intéressant, que l'on appellera ici «recatégorisation». On entend par cette notion un mouvement manifestement stratégique de justification des différends permettant de constater que la réalité juridique, dans une certaine mesure, autorise sa propre révision, son adéquation aux situations (Restrepo 2007 : 508, nous traduisons). On va s'efforcer de montrer que la recatégorisation est une stratégie très productive à la disposition des magistrats pour (ré)interpréter, voire revisiter la réalité juridique, au moins dans nos données.

10

Pour saisir ce qu'on entend par recatégorisation, on peut partir du concept de « dissociation » proposé par Perelman et Olbrechts-Tyteca. Dans le Traité, on le sait, les auteurs présentent la dissociation des notions comme un ensemble de «techniques de rupture ayant pour but de dissocier, de séparer, de désolidariser, de rompre, des éléments considérés comme formant un tout ou du moins un ensemble solidaire au sein d'un même système de pensée " (2008: 255). La dissociation exprimerait une «vision du monde » (ibid. : 561) en ce qu'elle établit une distinction dans «ce qui tend à être confondu ou associé » (Dominicy 2002 : 9), et tend à établir une hiérarchie entre les aspects ainsi distingués. Pour Perelman et Olbrechts-Tyteca, une fois la norme réévaluée par la dissociation, on dispose d'une "nouvelle vision du réel ", à travers laquelle on peut désormais juger les "nouveaux cas d'espèce »; et c'est précisément sur ce point que la notion de dissociation perelmanienne demande à être retravaillée pour rendre compte de nos données.

11 En effet, une raison pour préférer le concept de recatégorisation à celui de dissociation est liée au fait que, dans les données examinées, il ne nous semble pas que les juges qui distinguent l'idée d'« ennui » de celle de "vrai ennui/ fait illicite » comptent résoudre «un paradoxe, une contradiction». Ils se contentent de mobiliser stratégiquement cette distinction selon leur visée argumentative. En d'autres termes, dans le corpus dont on présentera ci-dessous un bref aperçu, cette scission (recatégorisation) est le fruit d'un choix des magistrats entre des catégories déjà existantes, et non le résultat d'une innovation conceptuelle et philosophique. Il semble que la disjonction opérée par les magistrats lorsqu'ils (re)catégorisent les faits a une visée essentiellement 
stratégique, pas nécessairement gouvernée par un besoin métaphysique de créer un nouveau concept pour exprimer une idée qui n'existait pas auparavant.

Le statut même de la dissociation des concepts soulevant encore des débats passionnants mais épineux ${ }^{4}$, on développera ici une notion plus modeste, mais mieux adaptée aux données dont on cherche à rendre compte. C'est pourquoi on parlera de « recatégorisation » plutôt que de « dissociation ».

\subsection{Le renverseur}

On le verra, la recatégorisation telle qu'on vient de l'évoquer est une façon efficace employée par les magistrats de présenter des arguments contraires à ceux de leurs pairs. Une telle dynamique (catégoriser X soit comme un $\mathrm{Y}$ soit comme un vrai $\mathrm{Y}$ ) est possible du fait que le raisonnement juridique prend en compte, simultanément, les valeurs, l'expérience vécue, ainsi que les normes et principes juridiques, dans la phase de qualification des affaires. Cet "élément d'incertitude» (= valeurs + expérience vécue + normes + principes) agirait comme une sorte de « dispositif » qui permettrait au magistrat de renverser la décision proposée par ses pairs dans le cadre des délibérés. A ce processus stratégique de séparation, c'est-à-dire de recatégorisation, s'ajoute une sorte d'outil argumentatif dont on montrera la mise en œuvre dans l'analyse qui suit. Il s'agit du renverseur (O), qui consiste en une justification fonctionnelle ${ }^{5}$ permettant aux magistrats de structurer et soutenir leurs avis, à leur gré, au moment des délibérés. La description de cet outil permet de montrer qu'une décision juridique s'éloigne vraiment du raisonnement mathématique, logique, exact. Avec son aide et grâce à la fluidité de la notion de « préjudice moral » au Brésil, il leur est possible de recatégoriser stratégiquement les faits, de façon à orienter leurs conclusions, antagoniques, aux moments où surgissent des stases argumentatives. On peut représenter ce processus de la façon suivante :

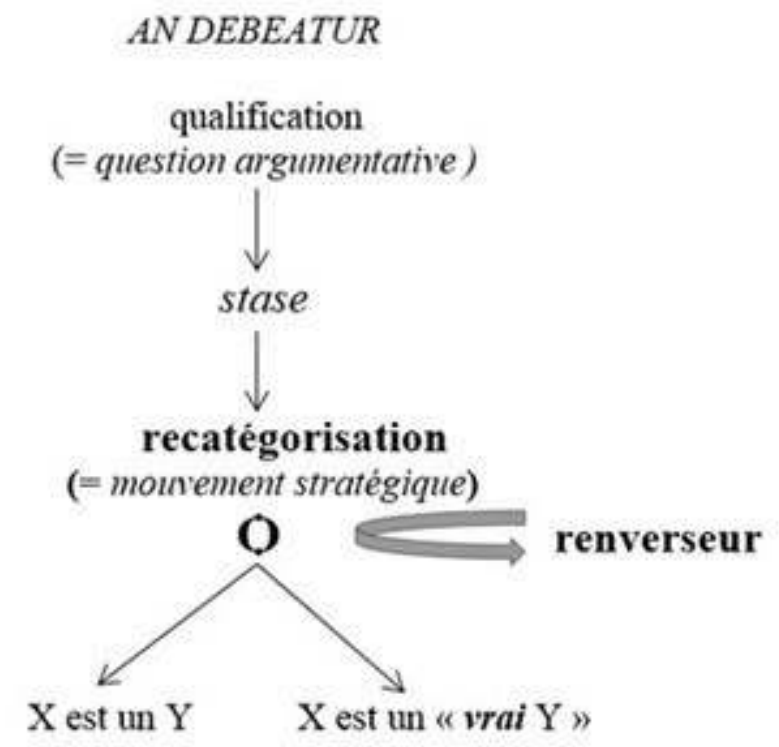

Figure 1: processus de recatégorisation

Selon le schéma ci-dessus, tout procès en appel active une question argumentative (Plantin 2005) du type «La plainte du demandeur implique-t-elle vraiment qu'il y a eu dommage moral ? » dans la première partie de chaque délibéré (dite an debeatur). Une 
fois la stase (au sens de conflit) établie, les magistrats devront justifier leurs décisions en recourant au mouvement stratégique de recatégorisation. En résumé, ce qu'on considère comme une opération de recatégorisation est rendue possible par une caractéristique majeure du domaine juridique: le fait de permettre au magistrat d'interpréter un certain fait de façons différentes, et toujours au nom de la loi. De cette façon, le dispositif argumentatif appelé renverseur $(O)$ participe du fait que la qualification « $\mathrm{X}$ est un $\mathrm{Y}$ » peut n'être que temporaire, car $\mathrm{Y}$ peut être interprété comme un « vrai $Y$ » (c'est-à-dire, comme une action «illicite») ou non, selon la visée argumentative du magistrat.

De cette façon, le renverseur qui symboliquement représente les valeurs, l'expérience vécue du magistrat, les normes et principes juridiques, permet au magistrat de faire un virage à $180^{\circ}$ dans une décision juridique avancée précédemment par un autre magistrat ${ }^{6}$. En résumé, la recatégorisation, telle qu'on l'envisage, est une façon que le juriste aurait d'adapter les circonstances de fait démontrées à la norme adoptée, étant donné que «l'ordre juridique dans une large mesure subsiste, s'alimente et se modifie grâce à sa dimension autoréflexive, qui lui confère la possibilité d'une réinterprétation continuelle de son dispositif normatif » (Stamakis $1995: 211$ ).

En partant du schéma ci-dessus, il nous reste à essayer de montrer comment, dans quelques affaires $\mathrm{du}$ corpus dont on dispose, se manifeste la stratégie de recatégorisation. Toutefois avant de développer l'analyse, il convient de préciser le contexte juridique brésilien qui a fourni les données.

\section{Le dommage moral et la procédure judiciaire brésilienne}

Dans le domaine du droit de la responsabilité civile, que ce soit dans la législation spécifiquement brésilienne ou dans le système juridique d'autres pays, la question du préjudice moral constitue un sujet épineux ${ }^{7}$. Il existe de nombreuses définitions du "préjudice moral » ainsi que diverses distinctions au sein de cette notion, du moins au Brésil. Dans cet article, le préjudice/dommage moral sera compris comme "une agression qui heurte l'intimité et entraîne une véritable affliction de l'esprit » (Reis $2010: 9)$.

18 La résolution des conflits qui sont jugés dans ce domaine peut impliquer le versement de dommages et intérêts d'un montant qui doit être déterminé lors d'une procédure civile, en réparation de préjudices moraux ou même physiques. La somme qui sera payée par l'offenseur s'appelle le pretium doloris; il n'est pas rare que son montant fasse l'objet de controverses, soit au moment de la qualification d'un acte comme "préjudice ", soit au moment de la définition des critères permettant d'établir le montant qui devra être versé en compensation d'une action considérée comme dommageable, selon les lois civiles du Brésil.

\subsection{Le corpus et le cadre participatif}

«L'affaire de la file d'attente » appartient au corpus TRIBUNAL, qui contient 64 affaires, enregistrées en audio et récoltés dans un tribunal brésilien. Toutes les affaires ont été jugées en appel entre 2000 et 2010. De ce corpus, on a sélectionné un cas dans lequel la 
gestion du désaccord entre les magistrats nous a semblé intéressante pour illustrer la recatégorisation argumentative.

La procédure judiciaire brésilienne en appel est publique, sauf pour les affaires définies comme relevant du "secret de l'enquête et de l'instruction». Selon la nature de l'affaire, la séance de délibération réunit trois ou quatre juges. Au début de chaque délibéré, le Juge Rapporteur expose son avis, déjà notifié aux autres magistrats par écrit avant la délibération, et qui énonce sa décision. Toutefois, en fonction du déroulement des délibérations, il peut changer d'avis et modifier la décision annoncée précédemment. Tout dépend du délibéré, qui se déroule oralement. Après l'énoncé de l'avis du Juge Rapporteur/RAP, le Premier Conseiller (C1), qui dans cette affaire exerce aussi la fonction de réviseur, est le deuxième juge à énoncer son appréciation. Cela implique que $\mathrm{C} 1$ a lui aussi deux possibilités : il peut se positionner soit en faveur, soit contre la proposition du Rapporteur. Le troisième juge, ou le Conseiller 2 (C2), qui en général ne connaît pas les éléments du procès avec autant de précision, prend position en se basant sur le récit fait par le Rapporteur. Il est le dernier à faire connaître son choix, avant l'annonce officielle du résultat des délibérations, qui sera proclamée par le Président de séance.

\section{L'affaire de la file d'attente : analyse}

21 L'affaire 7 traite du temps, considéré comme excessif, passé par un étudiant dans une file d'attente: venu à l'accueil de la faculté pour régler diverses questions, il a dû patienter quatre heures avant d'y accéder. Il faut préciser que, selon une loi brésilienne, toute personne qui reste plus de trente minutes dans une file d'attente a le droit de porter plainte en justice car, toujours selon cette loi, les établissements (banques, hôpitaux etc.) doivent faire en sorte que l'attente soit la plus brève possible. Cependant, cette loi est sujette à polémique, et de nombreux établissements ne la respectent pas, arguant, entre autres, de la difficulté qu'il y aurait à la mettre en pratique. Pour bien comprendre cette affaire, il faut avoir à l'esprit que le Rapporteur marque son accord avec la décision du Juge de Première Instance (ci-après JPI), qui ne voyait pas d'illicéité dans le cas considéré (il ne considère pas l'attente comme « illicite», mais comme un « simple ennui »). De leur côté, les deux Juges Conseillers expriment leur désaccord avec la position soutenue par le JPI. Cependant, comme les délibérations sont toujours composées de trois magistrats, les positions exprimées par les juges conseillers contre celle du rapporteur suffisent à renverser la décision du premier jugement (deux contre un). Ainsi, la nouvelle décision rendue en appel va considérer effectivement que l'attente dans la file représente un préjudice moral (donc, une action illicite) et que l'institution (le défendeur, donc l'université en l'occurrence) devra dédommager l'étudiant (le demandeur) pour être resté quatre heures à attendre avant de pouvoir accéder à l'accueil ; et ce malgré les raisonnements contraires du Rapporteur et du JPI.

Examinons le moment où le Rapporteur motive sa position qui, comme celle du jugement de première instance, ne considère pas l'attente comme un préjudice moral (un fait illicite) ${ }^{8}$.

TRIBUNAL 7 : Affaire de la file d'attente ${ }^{9}$

4. euh: éminents collègues je voudrais lire un extrait du vote

5. car bien que je comprenne l'avis de la chambre mais en effet 
6. je ratifie le positionnement du jpi \un moment je lis d'abord

7. le résumé et après une partie du vote euh: dédommagement à cause

8. de l'attente dans une file pendant quatre heures préjudice

9. moral pas prouvé simple ennui ou contretemps du

10. quotidien ne cara-caractérise pas préjudice extra-patrimonial

11. (.)décision demande rejetée sentence ratifiée\}

12. la simple attente dans une queue pour temps

13. considéré long ne caractérise pas un préjudice d'ordre moral

14. (.) bien que personne n'apprécie d'attendre pendant plus de

15. quatre heures ce simple fait désagréable ne suffit pas pour

16. générer un illicite d'ordre moral selon la

17. JURISPRUDENCE largement reconnue $\backslash$ je vais la

18. citer tout de suite simple ennui du quotidien n'oblige pas

19. le dédommagement extrapatrimonial demande rejetée sentence

20. ratifiée \#je veux lire une partie du vote\# ((toux)) ici c'est

21. la première partie \#ça n'intéresse pas\# le défendeur demande

22. l'indemnisation à cause d'une attente en file pour plus de

23. quatre heures \en effet le fait est bien attesté

24. néanmoins il n'est pas

25. raisonnable que cette simple attente soit considérée comme un

26. préjudice important pour qui que ce soit surtout dans son for intérieur il

27. est évident> que la longue attente dans une queue

28. est un épisode désagréable et insupportable $\backslash$ aucun

29. citoyen sain d'esprit n'apprécie d'attendre longtemps

30. dans une queue soit debout soit assis pour régler ses

31. affaires quotidiennes cependant on ne peut pas conclure que

32. cet ennui puisse caractériser un préjudice $<$ ((changement du

33. ton de la voix)) surtout un préjudice d'ordre

34. moral> (.) devant la complexité des actes de la vie

35. moderne tous les citoyens qui vivent en société risquent

36. d'avoir des ennuis éventuels il s'agit des événements

37. normaux de la vie et qu'on ne peut pas éviter on doit

38. les supporter \ce sont de petits préjudices de tous les jours

39. et qui en raison de leur caractère anodin ne sont pas

40. dédommagés (.) envisager des indemnisations dans toutes ces

41. mésaventures au-delà d'accabler encore le pouvoir judiciaire

42. déjà surchargé rendrait la vie

43. en société IMPOSSIBLE et indésirable \#un moment\# de cette

44. façon je suis convaincue que la simple attente dans une file même pendant quelques heures ne caractérise pas un préjudice

45. extra patrimonial

46. (.) le préjudice d'ordre patrimonial étant celui

47. qui afflige considérablement l'être humain dans ses valeurs

48. c'est-à-dire dans son for intérieur (.) il s'agit de douleur

49. psychique intense et qui porte atteinte au concept de dignité

50. humaine \néanmoins considérer que dans cette affaire il y a atteinte aux droits de la

51. personne signifierait vider le contenu de la 
52. doctrine même par rapport à l'importance de ces droits (.)

53. on n'est pas ici en train de soupeser la douleur de qui que

54. ce soit mais tout simplement on essaie de trier quels

55. épisodes du quotidien doivent être protégés juridiquement par rapport à la loi

des droits de la personne \de la

56. même façon on n'ignore pas qu'il serait inutile d'essayer

57. de prouver la douleur éprouvée parce que pour la

58. caractérisation d'un préjudice moral il suffit tout

59. simplement de constater un fait illicite capable de porter atteinte aux droits de la

60. personne cependant c'est justement dans

61. l'absence de fait illicite ou du supposé préjudice

62. probablement commis par le défendeur l'obstacle à la légitimation du préjudice moral (.)du reste il n'est pas

63. crédible que le simple fait de rater un cours soit

64. constitutif d'un préjudice à son encontre\

65. de cette façon en considérant l'absence de fait illicite

66. il est impérieux que le défendeur ne paie rien relativement à

67. l'indemnisation demandée (.) et par rapport à ce sujet je rajoute euh

68. beaucoup c'est-à-dire euh pour ne pas vous fatiguer je rajoute

69. UNE jurisprudence de la juridiction supérieure et ah et je

70. ratifie la sentence je rejette la demande du demandeur (.) je

71. condamne encore le demandeur au paiement des frais engendrés par le procès $\backslash$

D'abord, on voit que le Rapporteur, dès le début de son intervention, fait référence aux décisions déjà prises par la chambre («bien que je comprenne l'avis de la chambre mais », en 5) ; ce commentaire du Rapporteur fait référence à une autre affaire qui fait elle aussi partie du corpus TRIBUNAL, et qui avait été jugée par cette même chambre et par ces mêmes magistrats. Pour poursuivre l'analyse, on sélectionnera les principaux arguments du Rapporteur (ratifiés par C2) grâce auxquels il cherche à construire son positionnement contre l'idée de dommage moral dans cette affaire :

\begin{tabular}{|c|c|c|}
\hline \multicolumn{3}{|c|}{ Rapporteur/arguments } \\
\hline 1 & $\begin{array}{l}\text { " considérer que dans cette affaire il y a atteinte aux droits de la personne signifierait } \\
\text { vider le contenu de la doctrine même par rapport à l'importance de ces droits (.) \» }\end{array}$ & $\begin{array}{l}1 . \\
50-52\end{array}$ \\
\hline 2 & $\begin{array}{l}\text { «de la même façon on n'ignore pas qu'il serait inutile d'essayer de prouver la douleur } \\
\text { éprouvée parce que pour la caractérisation d'un préjudice moral il suffit tout } \\
\text { simplement de constater un fait illicite capable de porter atteinte [= préjudice] aux } \\
\text { droits de la personne\cependant c'est justement dans l'absence de fait illicite ou du } \\
\text { supposé préjudice probablement commis par le défendeur l'obstacle à la légitimation du } \\
\text { préjudice moral (.)» }\end{array}$ & $\begin{array}{l}1 . \\
55-62\end{array}$ \\
\hline 3 & $\begin{array}{l}\text { "(.) du reste il n'est pas crédible que le simple fait de rater un cours soit constitutif d'un } \\
\text { préjudice à son encontre \de cette façon en considérant l'absence de fait illicite il est } \\
\text { impérieux que le défendeur ne paie rien relativement à l'indemnisation demandée (.)\» }\end{array}$ & $\begin{array}{l}1 . \\
62-67\end{array}$ \\
\hline
\end{tabular}




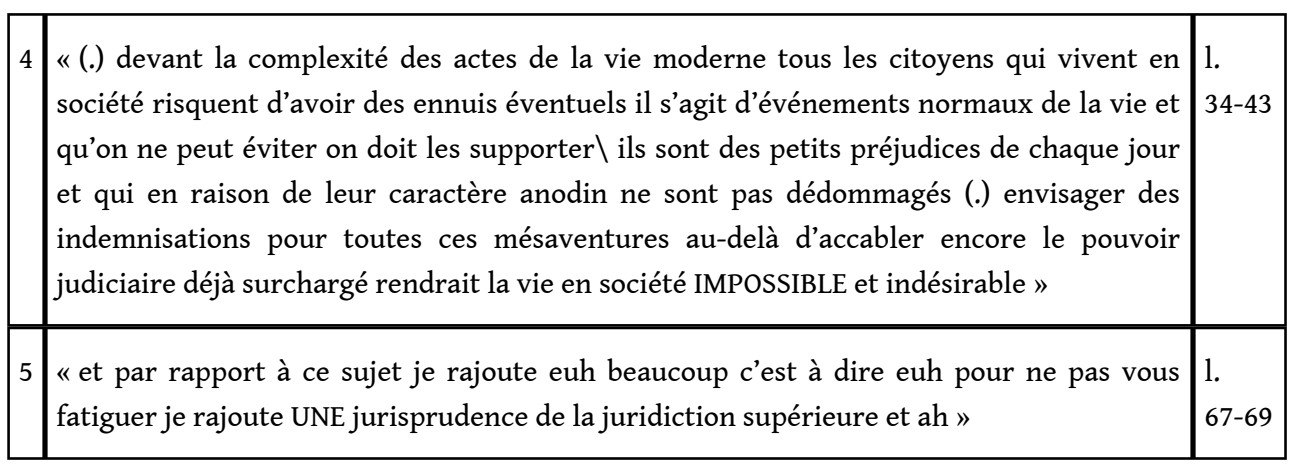

Si l'on se penche sur ces arguments et justifications, on voit que, de façon générale, le Rapporteur mobilise une parole technique pour construire une argumentation doctrinale (Livet 2000: 189). En regardant le tableau ci-dessus, on voit la matérialisation de la dimension juridique lorsque le juge rappelle la procédure en évoquant la doctrine, la preuve, un fait illicite capable de porter atteinte [ = préjudice] aux droits de la personne, le lien démonstratif etc. Par exemple, le quatrième argument est construit de façon à mettre en avant les conséquences plutôt négatives qu'aurait une décision contraire à la sienne: il s'agit d'un argument pragmatique (Perelman 1989), ou même de l'argument de la pente glissante, adossé à l'évocation du caractère catastrophique que prendrait ce genre d'affaire dans le cas d'une décision contraire à celle proposée par le Rapporteur. Cette façon de dépeindre les conséquences négatives accroît l'efficacité rhétorique de la parole du juge. L'argument 5 pourrait être caractérisé comme un argument par le précédent, dans la mesure où, quand le Rapporteur cite un argument tiré de la jurisprudence, il cherche un jugement similaire qui aurait été rendu antérieurement par une instance supérieure pour légitimer sa propre thès $\mathrm{e}^{10}$; il peut être considéré également comme un exemple de l'utilisation de la règle de justice (ou argumentation a pari), selon laquelle des cas semblables doivent subir des traitements analogues. Finalement, tout le chemin argumentatif parcouru par le juge rapporteur vise la conclusion suivante :

Rapporteur/conclusion

«de cette façon je suis convaincue que la simple attente dans une file même pendant 1 .

quelques heures ne caractérise pas un préjudice extra patrimonial \»

Notre but n'étant pas de faire un catalogue des arguments utilisés, on s'en tiendra essentiellement au fait que, à partir du refus de considérer une longue attente dans une file comme constitutive d'un préjudice moral, le Rapporteur fait une distinction entre action illicite aux yeux de la justice versus action légale, mais "déplaisante et ennuyeuse» pour celui qui la subit. De cette façon, il mobilise une dichotomie structurée autour de deux catégories à partir de la notion floue (selon les magistrats brésiliens) de "dommage ». On voit que le Rapporteur réfute la thèse de "l'illicéité " défendue par la partie plaignante et soutient la thèse du "simple ennui ", selon laquelle les simples ennuis éprouvés par le demandeur ne sont que « des événements normaux de la vie et qu'on ne peut pas éviter » (1.36-37). 


\subsection{La recatégorisation à vif et à chaud}

72. C1 je demande la permission à l'honorable juge

73. rapporteuse

74. car lors d'un vote antérieur proféré devant cette chambre où : pour une question moindre j'avais ratifié

75. l'illicite

dans une affaire à propos de longue attente dans une queue

76. dans une banque je vois qu'il s'agit d'une

77. institution qui offre plus de quarante cours de droit partout

78. dans le Brésil $\backslash$ une institution présente dans presque toutes

79. les villes brésiliennes et par expérience de vie $\backslash$ en m'

80. appuyant sur l'article trois cents trente-cinq du code civile je suis convaincu que la longue

81. attente dans une file d'accueil est due à la négligence et

82. au désintérêt de la défenderesse à l'égard de ses étudiants \c'est-à-dire la grande quantité d'étudiants inscrits et qui

83. contraste avec l'insuffisance de personnel à l'accueil

84. les gens qui s'occupent du secrétariat changent presque tous les mois (.) je connais très bien cette situation je

85. suis ex

86. employé et ex professeur de la défenderesse et cela ne

87. m'empêche pas de prononcer mon vote maintenant

88. parce que je n'ai plus aucun rapport avec cette institution depuis très longtemps $\backslash$ je connais très bien cette

89. situation je le redis et

90. réellement c'est une situation difficile dans tous les cours

91. ou des facultés ou universités dans le ((nom de la ville)) cette question des élèves par rapport à l'effet pédagogique

92. punitif didactique

93. et l'effet que situations comme celles-là ((incompréhensible) dans cette institution au moins pour ceux qui restent en

94. dehors (chaises) ((on ne comprend pas même en portugais!))

95. désormais mettre à disposition chaises et une télé je préviens et je connais de très près cette situation

96. il n'y a pas de raison de ne pas caractériser comme préjudice mora:l l'inconvénie:nt la longue attente de

97. l'élève dans la file

98. comme bien rapporté par l'illustre rapporteuse \l'élève

99. d'une certaine façon a raté un cours on ne sait 
100. pas exactement pour quelle raison mais évidemment en tant que exprofesseur de cette institution il y était commun le

101. retard des élèves même les jours de test

102. dont la justification était le retard dans la file d'attente

103. où ils essaient de régler des affaires administratives (.)

104. de cette façon et en considérant l'effet pédagogique préventif et punitif oui je considère qu'il y a préjudice moral et qu'il existe une infraction à la loi de

105. protection du consommateur car le préjudice est in re ipsa

106. c'est-à-dire il n'y a pas la nécessité de la preuve la

107. défenderesse même avoue que les files existent

108. et que le demandeur était resté dans la queue le fait n'est

109. pas controversé dans ce sens je vote pour le revirement de

110. la sentence et je propose à titre de dédommagement deux mille

111. reais ce qui ne produit pas d'enrichissement sans cause à

112. personne surtout au-au défendeur mais c'est pour que cela génère un effet pédago:gique et punitif voilà mon vote

On note que, après avoir présenté ses excuses (1.72), le premier juge-conseiller évoque, lui aussi, un jugement antérieur sur le même sujet en utilisant une justification du type a maiori ad minus (1.73-75). De cette façon, le magistrat fait état des arguments qui, selon lui, justifient son désaccord par rapport au raisonnement avancé par le Rapporteur et qui marque le durcissement de la stase entre eux. Le tableau ci-dessous récapitule les contre-arguments employés par $\mathrm{C} 1$ :

\begin{tabular}{|l|l|l|}
\hline \multicolumn{2}{|l|}{ Premier conseiller/arguments } \\
\hline 1 & $\begin{array}{l}\text { «cette question des élèves par rapport à l'effet pédagogique punitif didactique a pour } \\
\text { effet que situations comme celles-là n'existent pas dans cette institution » }\end{array}$ & $1.91-93$ \\
\hline 2 & $\begin{array}{l}\text { «le préjudice est in re ipsa c'est-à-dire il n'y a pas la nécessité de la preuve la } \\
\text { défenderesse même avoue que les files existent et que le demandeur était resté dans la } \\
\text { queue» }\end{array}$ & $104-107$ \\
\hline 33 & «pour que cela génère un effet pédago :gique et punitif» & 1.112 \\
\hline 4 & $\begin{array}{l}\text { «je propose à titre de dédommagement deux mille reais ce qui ne produit pas } \\
\text { d'enrichissement sans cause à personne surtout au-au défendeur » }\end{array}$ & 1. \\
\hline
\end{tabular}

Le premier juge-conseiller énonce des contre-arguments qui renforcent l'idée soutenue par quelques juristes, selon lesquels le plaignant n'a pas besoin de prouver sa souffrance, et qu'il suffit de prouver l'illicéité du cas, ainsi qu'il le dit dans les contrearguments 2 et 4 . Les autres contre-arguments ( 1 et 3 ) sont liés aux critères communément utilisés par les magistrats au moment de définir le pretium doloris. Après avoir fait état de ses arguments, le premier conseiller finit son raisonnement sur la conclusion suivante :

Premier conseiller/conclusion 
\begin{tabular}{|l|l|}
\hline «je considère qu'il y a préjudice moral (...) » & 1.104 \\
« dans ce sens je vote pour le revirement de la sentence $\backslash »$ & 1.108 \\
\hline
\end{tabular}

31 On dégage de cette façon deux raisonnements qui aboutissent à des conclusions opposées. D'un côté, le Rapporteur (comme le JPI) ne considère pas l'événement de l'attente excessive dans une file comme un préjudice moral, mais comme un simple ennui de la vie quotidienne. De l'autre côté, les deux conseillers, à l'inverse, soutiennent qu'il ne s'agit pas d'un "simple ennui» mais d'une fait illicite ayant engendré un préjudice moral. Chaque magistrat, selon sa propre visée argumentative, justifie le jugement « $\mathrm{X}$ est un $\mathrm{Y}$ » à partir d'une recatégorisation particulière.

On a pu montrer aussi que, dans chacun des raisonnements analysés ci-dessus, chaque magistrat offrait stratégiquement une version différente d'un même événement. Ainsi, on voit que les magistrats partent d'une notion floue ( = dommage) pour qualifier l'affaire selon leur propre visée argumentative, qualifiant l'affaire de la façon la mieux à même de soutenir leurs arguments individuels. De cette façon, on s'éloigne du concept perelmanien de "dissociation de concepts » car ici on ne procède à aucune recomposition de concepts philosophiques. On observe plutôt la façon dont la visée argumentative oriente les raisonnements des magistrats vers des conclusions antagonistes dans une situation de stase. Le schéma ci-dessous met en évidence le procédé de recatégorisation et montre comment les magistrats scindent l'idée générale de « dommage », à partir des arguments identifiés dans les tableaux ci-dessus :
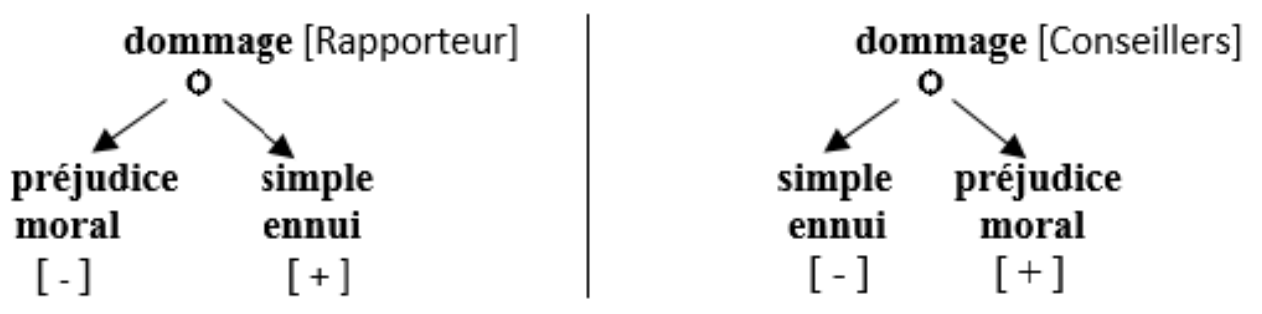

Figure 2 : Le renverseur en action

Ces schémas nous montrent d'une façon résumée le type de raisonnement exposé par les magistrats. D'abord, le Rapporteur soutient des arguments qui montrent que l'épisode de la file d'attente n'est pas constitutif d'un préjudice moral. En faisant référence à la loi, ce magistrat commence par catégoriser l'affaire en essayant de réfuter/dévaloriser [-] l'idée de dommage ; il essaye de la discréditer en faisant état des arguments résumés plus haut. Ensuite, ce juge, en conclusion de l'exposé de ses motifs, valorise l'idée de simple ennui [ + ], et la visée argumentative de la manœuvre devient alors évidente, puisque selon ce magistrat, l'attente dans la file ne serait pas illicite. De l'autre côté, le juge-conseiller va dans le sens inverse: il recatégorise le même événement, suivant cette fois-ci le chemin opposé à celui proposé par le Rapporteur. En effet, il réfute [ - ] l'idée de « simple ennui » et soutient/valorise [ + ] la thèse du «préjudice moral » afin d'aboutir à la conclusion inverse. Le symbole $O$, rappelons-le, marque le moment où le magistrat essaie de renverser le raisonnement soutenu par son confrère. Le renverseur représenterait le déclencheur de la recatégorisation opérée par le magistrat antagoniste. 0 matérialise également les éléments d'incertitude dont on a parlé, dans la première partie de cet article, où l'on 
a admis le fait qu'il est possible pour le magistrat d'appuyer ses décisions sur des valeurs, sur l'expérience vécue, les normes, les principes. Ce symbole représente, en fin de compte, et d'une façon simplement illustrative, les revirements de décisions dans le domaine juridique, comme on vient de le montrer.

\subsection{L'expérience personnelle comme déclencheur de la recatégorisation}

Le tour de parole par lequel le premier juge-conseiller manifeste son désaccord vis-àvis de l'opinion qui vient d'être prononcée par le juge rapporteur met à jour la subjectivité des magistrats. En effet, on a observé que le Conseiller s'exprime de façon très catégorique lors de sa contre-argumentation transcrite ci-dessous et qu'il connaît de «très près » cette situation. En fait, le magistrat répète cette information à maintes reprises, comme on le voit dans le tableau suivant :

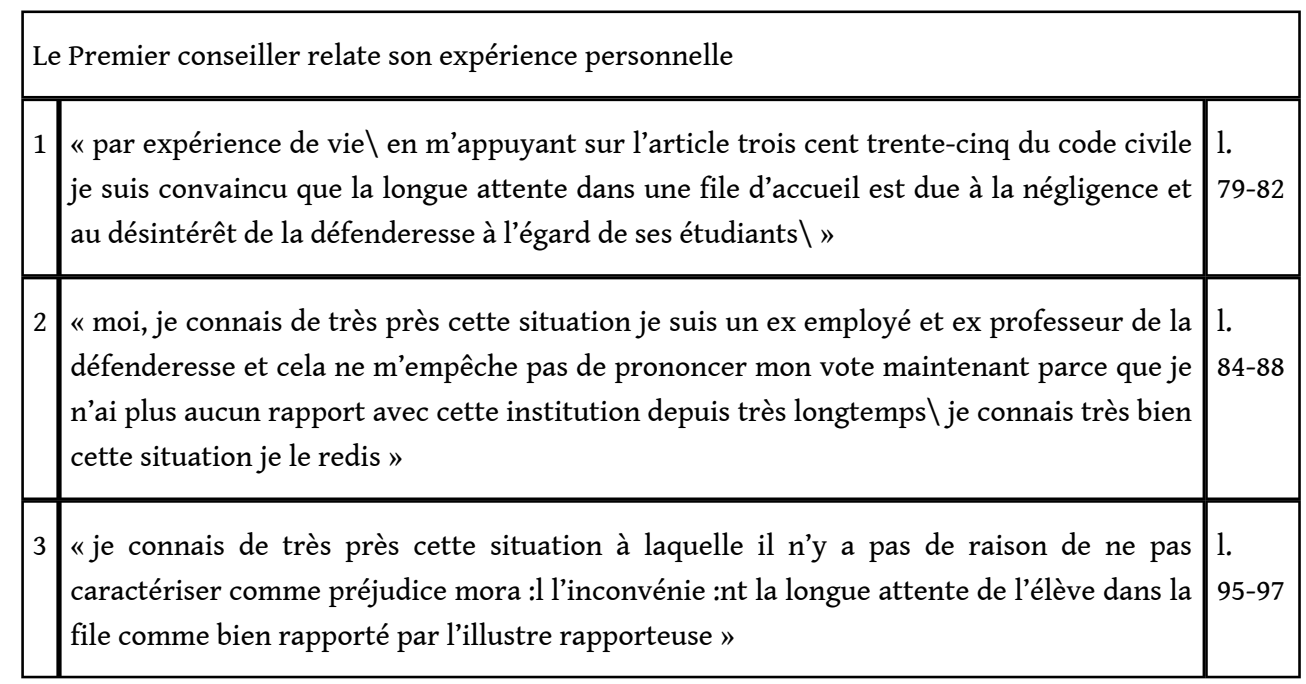

On a constaté, dans la première partie de l'analyse de cette affaire, que le jugeconseiller, au moment de contre-argumenter, recourt à des arguments techniques du domaine du droit ; cependant, dans cet autre extrait, le même magistrat, au moment de réfuter la conclusion proposée par le Rapporteur, met en avant lors de sa prise de parole son expérience vécue et personnelle en tant que professeur, en tant qu'individu. Cette façon de soutenir sa contre-argumentation en thématisant sa personne suggère un certain investissement personnel (Amossy 2010) du locuteur: «Moi, je connais de très près cette situation ». On fait l'hypothèse que ce " témoignage » aurait en fait une fonction stratégique, car cette parole finit par orienter la conclusion du magistrat, laquelle s'oppose à la conclusion du Rapporteur. C'est pourquoi on soutient que cette parole a une valeur nettement argumentative : elle aide le premier juge-conseiller à soutenir son positionnement antagoniste, ce qui maintient la stase, le moment conflictuel, lors du délibéré. Cela montre bien que le magistrat est loin de l'image du juge froid, « juge-machine », alexithymique ${ }^{12}$, image portée par la philosophie légaliste du droit. On observe que le juge s'expose en tant qu'individu dans ses paroles, d'une façon proche de la confidence et, plus surprenant encore, qu'il l'avoue. En réalité, le raisonnement construit par $\mathrm{C} 1$ montre que son expérience personnelle devient un 
« outil » important dans l'élaboration de sa décision dans cette affaire. Selon Grize (1996 : 43),

la dimension du vécu est tout spécialement importante et ceci pour deux raisons. D'abord elle enrichit la signification par tout un ensemble de connotations qui jouent un rôle non négligeable dans l'argumentation et ensuite elle marque la différence entre les sujets humains et les ordinateurs.

Cela ne signifie évidemment pas que son raisonnement soit biaisé, fallacieux ou truqué, ainsi que le spécifie le magistrat lui-même (« et cela ne m'empêche pas de prononcer mon vote maintenant parce que je n'ai plus aucun rapport avec cette institution depuis très longtemps \» 1.85-88). Intervient ici l'idée de "désintéressement » (et non de désintérêt) : il se peut que le magistrat s'intéresse bel et bien au débat, sans que cela signifie pour autant que sa décision soit déterminée par ses intérêts personnels (Garapon 2008 : 36).

De cette façon, quand $\mathrm{C} 1$ « atteste » avoir connu « de très près » ce type d'épisode (la longue attente dans une file), il suggère que cette situation est caractéristique d'une véritable illicéité ; son " témoignage ", son expérience personnelle, son "vécu », sont employés comme le déclencheur de son désaccord envers des collègues magistrats. On considère que l'expérience personnelle avouée par le magistrat, dans ce moment, fonctionne comme le renverseur $(O)$ qui déclenche la recatégorisation [ennui (licite) vs vrai ennui (illicite)] d'un même fait (l'attente), ce qui, en fin de compte, a permis à ce magistrat de faire triompher son point de vue, c'est-à-dire, sa propre catégorisation d'un fait dans le domaine juridique.

\section{Conclusion}

Le parcours analytique commence, au début de l'article, par la description de la recatégorisation comme mouvement stratégique de justification des différends. On a étudié la manière dont les magistrats allient la liberté et la raison au moment de qualifier un fait en tant que « dommage moral » - concept ouvert à maintes possibilités d'interprétation juridique au Brésil. On s'est efforcé de montrer que cette recatégorisation est un procédé productif auquel recourent les magistrats pour (ré)interpréter, réviser la réalité juridique. Ainsi, l'identification de ce qu'on a appelé « renverseur » $(O)$, c'est-à-dire le dispositif qui permet aux magistrats de changer une décision, nous aide à comprendre comment les avis des juges peuvent s'affronter catégoriquement. Ces observations confirment qu'une décision juridique s'éloigne vraiment du raisonnement mathématique, logique, exact. Et finalement on pourra considérer cette étude comme un exemple de la forme que peut prendre la gestion du désaccord en cas de conflit d'opinions entre magistrats dans une Cour d'Appel. 


\section{BIBLIOGRAPHY}

Amossy, Ruth. 2010. La présentation de soi - Ethos et identité verbale (Presses Universitaires de France)

Angenot, Marc. 2008. Dialogues de sourds - traité de rhétorique antilogique (Mille et une Nuits)

Cornu, Gérard. 2005. Linguistique juridique (Paris : Montchrestien)

Danblon, Emmanuelle. 2002. Rhétorique et rationalité - Essai sur l'émergence de la critique et de la persuasion (Éd. de l'Université de Bruxelles)

Dominicy, Marc. 2002. « La dimension sémantique du discours argumentatif : le travail sur les notions », Roselyne Koren \& Ruth Amossy (éds). Après Perelman. Quelles politiques pour les nouvelles rhétoriques? (Paris : L'Harmattan)

Dupret, Baudoin. 2006. Le jugement en action - ethnométhodologie du droit, de la morale et de la justice en Egypte (Genève \& Paris : Droz)

Feteris, Eveline T. 1999. Fundamentals of legal argumentation - A survey theories on the justification judicial decisions (Netherlands: Kluwer Academic Publishers)

Garapon, Antoine. 2001. Bien juger. Essai sur le rituel judiciaire (Paris : Odile Jacob)

Grize, Jean-Blaise. 1996. Logique naturelle et communication (Collection Psychologie Sociale. Presses Universitaires de France)

Hiltunen, Risto et al. 2010. Discourse and context in a historical perspective - Discourses in interaction (Amsterdam/Philadelphia : John Benjamins)

Livet, Pierre (éd.). 2000. L'argumentation: droit, philosophie et sciences sociales (Canada : Presses universitaires de l'Univ. Laval)

Martineau, François. 2010 (4e éd.). Petit traité d'argumentation judiciaire (Paris : Dalloz)

Meyer, Michel. 1999. Histoire de la Rhétorique des Grecs à nos jours (Librairie Générale Française)

Perelman, Chaïm. 1989. Rhétoriques (Belgique : Éd. de l’Université de Bruxelles)

Perelman, Chaïm. 1990. Éthique et droit (Belgique : Éd. de l'Université de Bruxelles)

Perelman, Chaïm (1999). Logique juridique - Nouvelle rhétorique (Paris : Dalloz)

Perelman, Chaïm \& Lucie Olbrechts-Tyteca. 2008. Traité d'argumentation (Belgique : Éd. de l'Université de Bruxelles)

Plantin, Christian. 2005. L'argumentation (Paris : PUF - Que sais-je ?)

Plantin, Christian. Dictionnaire de l'argumentation (à paraître)

Posner, A. Richard. 2008. How judges think (London: Harvard University Press)

Reis, Clayton. 2010. Dano moral (Rio de Janeiro : Forense)

Restrepo, Gabriel Mora. 2007. « La estrutura del saber jurídico ». La argumentation jurídica Compilatión y extratos. Fernando Quiceno Álvarez (Colombia : Ed. Jurídica)

Stamakis, Constantin M. 1995. Argumenter en droit - une théorie critique de l'argumentation juridique (Paris : Publisud)

Vignaux, Georges. 1979. « Argumentation et discours de la norme », Langages $12:$ 53, 67-85 


\section{NOTES}

1. Selon Plantin, l'idée de stase naît d'une notion technique issue de l'univers de la médecine, qui traduit le blocage de la bonne circulation des fluides humains et qui, transposée au monde de l'argumentation et de l'interaction, renvoie au conflit, au désaccord, aux différends et divergences entre parties lors d'une discussion (Dictionnaire de l'argumentation - à paraître -, art. Stase).

2. L'expression «à vif et à chaud " a été empruntée à Cornu (2005:252), quand il présente les caractéristiques du débat oral.

3. Ici les mots illicite/illicéité sont toujours employés dans le sens de «fait dommageable », "fait à l'origine du dommage » ou encore «fait générateur du dommage », pour éviter des polémiques liées à des questions juridiques de terminologie.

4. Gâta, A. (2011); Muraru, D. (2011) : Proceedings of 7o ISSA/Amsterdan; Konish, T. (2003) Proceedings of 50 ISSA/Amsterdan.

5. Pour Livet les «justifications fonctionnelles (opposées aux justifications doctrinales) utiliseront des éléments juridiques, mais sans pouvoir assurer que le choix de ces éléments juridiques sera lui-même d'origine juridique » (2000: 189).

6. Et c'est pour cette raison qu'on utilise le symbole $O$, lequel donne l'idée de mouvement, ou plus exactement de rotation.

7. Les livres suivants expliquent la question de la fluidité du concept de "préjudice moral » au Brésil : Clayton Reis, Dano Moral, 2010 ; Yussef Said Cahali, Dano Moral, 2005 ; Eduardo de Oliveira Leite, Grandes Temas da Atualidade: Dano Moral, 2002 ; M. Francisca Carneiro, Avaliação do Dano Moral, 1998.

8. Pour une raison d'espace, on ne présente pas la version originale. Quant aux extraits en français, ils ne doivent pas être considérés comme des traductions précises des transcriptions originales (en portugais), on a simplement essayé de rendre compréhensibles l'original. Il s'est souvent avéré impossible de transcrire mot à mot ce que les magistrats disaient: il s'agit de discours oraux, qui sont parfois presque incompréhensibles même dans la langue originale.

9. Les conventions de transcription adoptées sont les suivantes: / intonation montante, I intonation descendante, (.) pause brève, (..) pause moyenne, (...) pause longue, (0.6) pause en seconds, [ ] chevauchement, xxx segment inaudible, ((rit)) commentaire, ( ) transcription incertaine, \& absence d'intervalle entre deux tours, = continuation du même tour, VIdéo emphase, : allongement, - interruption, ${ }^{\circ}{ }^{\circ}$ voix basse, $<(())$ bla bla ${ }^{`}$ \# \# voix accélérée.

10. Et il nous semble tenir également de l'argument d'autorité.

11. Cette parole est encore plus difficile à comprendre que la parole prononcée par le Rapporteur.

12. Selon le Dictionnaire de Plantin (à paraître), «Le terme alexithymie (a-lexis-thymos: manque de mots pour l'émotion) désigne un langage d'où est bannie toute expression des sentiments et des émotions ".

\section{ABSTRACTS}

In this study, we seek to understand and describe how disagreement between judges is handled in a deliberation on a given case in the context of a Brazilian Court of Appeal. Using a transcript of a trial dealing with moral damage (or the so-called "price of pain"), and drawing on the field of 
argumentation, legal argumentation and rhetoric, we examine the mechanisms of the "argumentative recategorization" in a conflict of opinion between judges. The analysis shows that, in addition to the precepts of law that are necessary to justify a decision, real-life experience may play a role in driving one interlocutor to adopt a decision that contradicts a decision already taken in the first instance. The brief analysis of the "Queue case » will allow us to see more clearly what jurists and philosophers of the legal field, such as C. Perelman, had already emphasized: in the legal field, a decision can be reversed and another solution adopted through argumentation.

Cette étude de cas cherche à comprendre et décrire quelques modalités de gestion du désaccord entre magistrats dans une situation argumentative polilogale, dans une Cour d'Appel brésilienne. En partant de la transcription d'un jugement concernant la réparation d'un préjudice moral, et en se basant sur certaines lectures issues du domaine de l'argumentation, de l'argumentation juridique et de la rhétorique, on cherchera à examiner le fonctionnement de ce que nous appelons «recatégorisation argumentative» quand émerge un conflit d'opinions entre les magistrats. L'analyse cherche à démontrer que, au-delà des préceptes de loi nécessaires à la justification d'une décision, l'expérience vécue jouera un rôle très important au moment du revirement d'une décision juridique plus ancienne, prise en première instance. L'analyse de l'Affaire de la "file d'attente » nous permettra de voir plus clairement ce que les juristes et les philosophes du domaine juridique, comme C. Perelman, soulignaient déjà : sur le territoire juridique, une décision n'est jamais le résultat d'un calcul et, à cause de cela, en général on pourra, en argumentant, changer une solution, dès qu'on trouve la meilleure façon de le faire.

\section{INDEX}

Mots-clés: cour, désaccord, expérience vécue, préjudice moral, stratégie

Keywords: court, disagreement, lived experience, moral damage, strategy

\section{AUTHOR}

\section{RUBENS DAMASCENO MORAIS}

Université Lumière Lyon 2 